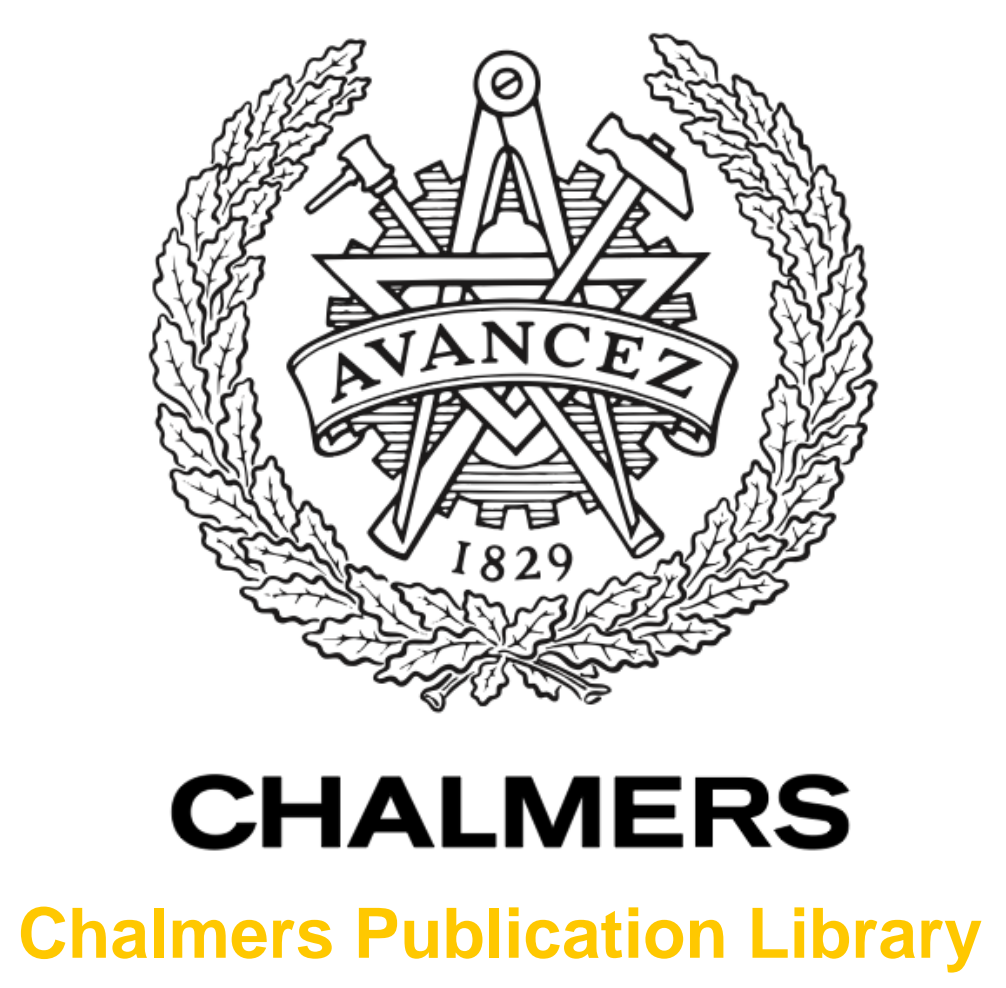

\title{
Performance bounds for multipath-assisted indoor localization on backscatter channels
}

This document has been downloaded from Chalmers Publication Library $(\mathrm{CPL})$. It is the author's version of a work that was accepted for publication in:

IEEE National Radar Conference: 2014 IEEE Radar Conference, RadarCon 2014; Cincinnati, OH; United States; 19 May 2014 through 23 May 2014 (ISSN: 1097-5659)

Citation for the published paper:

Leitinger, E. ; Meissner, P. ; Fröhle, M. (2014) "Performance bounds for multipath-assisted indoor localization on backscatter channels". IEEE National Radar Conference: 2014 IEEE Radar Conference, RadarCon 2014; Cincinnati, OH; United States; 19 May 2014 through 23 May 2014 pp. 70-75.

http://dx.doi.org/10.1109/RADAR.2014.6875557

Downloaded from: http://publications.lib.chalmers.se/publication/202625

Notice: Changes introduced as a result of publishing processes such as copy-editing and formatting may not be reflected in this document. For a definitive version of this work, please refer to the published source. Please note that access to the published version might require a subscription. 


\title{
Performance Bounds for Multipath-assisted Indoor Localization on Backscatter Channels
}

\author{
Erik Leitinger*, Paul Meissner*, Markus Fröhle ${ }^{+}$, and Klaus Witrisal ${ }^{*}$ \\ * Email: erik.leitinger@tugraz.at, Graz University of Technology, Austria \\ + Email: frohle@chalmers.se, Chalmers University of Technology, Sweden
}

\begin{abstract}
In this paper, we derive the Cramér-Rao lower bound (CRLB) on the position error for an RFID tag localization system exploiting multipath on backscatter radio channels. The backscatter channel is modeled with a hybrid deterministic/stochastic channel model. In this way, both the geometry of the deterministic multipath components (MPCs) and the interfering diffuse multipath are taken into account. Computational results show the influence of the room geometry on the bound and the impact of the diffuse multipath. Time reversal (TR) processing on the uplink channel is analyzed using the deterministic MPCs to overcome the degenerate nature of the backscatter channel. The CRLB shows the potential gain obtained from TR processing as well as its strong dependence on the geometry. Such TR processing has been proposed for $\mathrm{TX}$ waveform adaptation in the perception-action cycle of a cognitive radar. The results of this paper illustrate that it can indeed influence beneficially the measurement noise of the received signal, yielding control over the localization system.
\end{abstract}

\section{INTRODUCTION}

Ultra-wideband signals are promising candidates for localization in harsh indoor multipath environments, due to fine time-resolution enabled by large bandwidth. Nevertheless, indoor positioning is still a challenging task, in particular due to errors caused by non-line-of-sight (NLOS) propagation conditions. These problems are even stronger on backscatter channels employed by RFID systems.

Performance bounds such as the Cramér-Rao lower bound (CRLB) can yield valuable insights on the influence of the channel parameters on the localization accuracy. In [1], [2] the CRLB given by the information inequality is derived directly from the received signal rather than from specific features extracted from the signal. Multipath components (MPCs) associated to strong reflections can increase accuracy of the position estimation, if prior floor-plan knowledge is available [3]. These MPCs can be seen as originating from so-called virtual anchors (VAs) as shown in Fig. 1, hence a deterministic model is available for these MPCs. A key difference of [3], compared to [1], [2], is the stochastic modeling of the diffuse multipath (DM) which acts as interference for these useful geometryrelated components. The theoretical results from [3] were verified using data from an extensive indoor measurement campaign [4]. For conventional "non-backscatter" channels, the feasibility of multipath-assisted tracking algorithms was demonstrated in [5] for real channel measurements.

This work was partly supported by the Austrian Science Fund (FWF) within the National Research Network SISE project S10610.

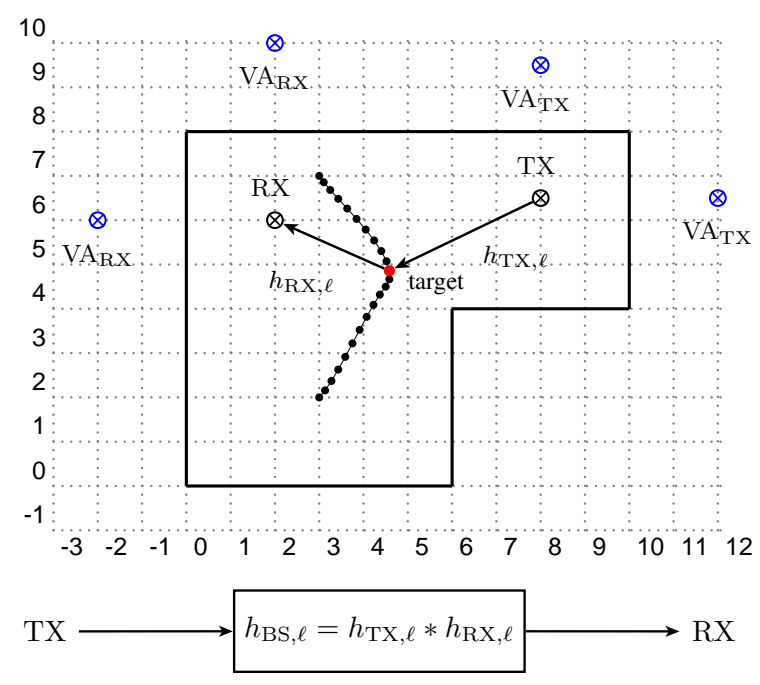

Fig. 1. Top: Floorplan with a transmitter (TX) and receiver (RX) radar node and a subset of corresponding VAs. The up-link is the channel between TX and the target at position $\ell$ along a trajectory, the down-link is the channel between the target and RX. Bottom: Backscatter model as concatenation of up- and down-link channels.

The contribution of this paper is the extension of the theoretical framework in [3] to the localization error bound of a radar target using a backscatter channel model. It is well-known that the channel in backscatter radio systems is a degenerate pinhole channel [6], formed by the concatenation of the channels from the transmitter (TX) to the target and from the target to the receiver (RX). In our work we assume that the signal received through the RFID tag can be distinguished from the signal coming directly from the environment, due to the modulation of the RFID tag.

Time-reversal processing (TR) [7], [8] for backscatter channels has been motivated in [9], to focus the energy onto RFID tags at certain positions and to separate the up- and downlink channels from one another. We analyze the impact of TR processing on the CRLB for multipath-assisted RFID localization in this paper. Common TR processing uses the entire reversed complex conjugate channel. This is not resilient w.r.t. imperfect channel knowledge, as any errors cause incoherent summation of paths and thereby loss of focusing of the energy. Therefore, we only use a limited set of deterministic MPCs that can be modeled geometrically, as these have been shown to carry a large fraction of the channel energy [4]. Such TR processing has been proposed for TX processing in the 
perception-action cycle of a cognitive radar [9]. Quantifying its impact on the CRLB provides a way to close this feedback loop.

This paper is organized as follows: The signal and channel models are given in Section II. The derivation of the CRLB and the evaluation of the influence of TR processing are provided in Section III. Results are discussed in Section IV. Finally, conclusions are drawn.

\section{Signal And Channel Models}

In order to give a mathematical description of the received signal $r(t)$, which is needed to compute the CRLB, we first introduce a model for a hybrid, deterministic/stochastic channel [3] and secondly for a backscatter channel as the concatenation of the former ones.

\section{A. Hybrid Deterministic/Stochastic Channel}

The hybrid deterministic/stochastic complex baseband radio channel $h_{m, \ell}(\tau)$ between a radar node $m \in\{\mathrm{TX}, \mathrm{RX}\}$ located at position $\mathbf{p}_{1, m}$ and a target at position $\mathbf{p}_{\ell}$ is defined as

$$
h_{m, \ell}(\tau)=\sum_{k=1}^{K_{m, \ell}} \alpha_{k, m, \ell} \delta\left(\tau-\tau_{k, m, \ell}\right)+\nu_{m, \ell}(\tau)
$$

where the first term consists of $K_{m, \ell}$ deterministic MPCs with complex amplitudes $\alpha_{k, m, \ell} \in \mathbb{C}$ and delays $\tau_{k, m, \ell}=$ $\frac{1}{c}\left\|\mathbf{p}_{\ell}-\mathbf{p}_{k, m}\right\|$, where $c$ is the speed of light. The delays of deterministic MPCs, i.e. strong specular reflections on walls, can be modeled geometrically, if the floor-plan is known. Fig. 1 shows the locations $\left\{\mathbf{p}_{k, m}=\left[x_{k, m}, y_{k, m}\right]^{T}\right\}_{k=1}^{K_{m, \ell}}$ of a subset of VAs in the considered environment. The second term $\nu_{m, \ell}(\tau)$ denotes the diffuse multipath (DM) and is modeled with a stochastic process. We assume uncorrelated scattering (US), so that the ACF of the DM is given as

$$
K_{\nu}(\tau, u)=\mathbb{E}\left\{\nu_{m, \ell}(\tau) \nu_{m, \ell}(u)^{*}\right\}=S_{\nu, m, \ell}(\tau) \delta(\tau-u) .
$$

$S_{\nu, m, \ell}(\tau)$ is the power delay profile (PDP), where $S_{\nu, m, \ell}(\tau)=$ 0 for $\tau<\tau_{1, m, \ell}$, which implies that the DM does not exist until the first deterministic MPC excites the channel. For a specific radar node $m$ and a well-defined "local area" around the target position $\mathbf{p}_{\ell}$ (several wavelengths), DM is assumed to be quasi-stationary, which means that the channel's first- and second-order statistics do not change noticeably in the spatial domain [10].

\section{B. Backscatter Channel}

For brevity, we drop the tag position index $\ell$ in further derivations. The backscatter channel impulse response (CIR) is obtained by the convolution of the up- and down-link channels both modeled with (1) yielding

$$
\begin{aligned}
& h_{\mathrm{BS}}(\tau)=h_{\mathrm{TX}}(\tau) * h_{\mathrm{RX}}(\tau) \\
& =\sum_{k=1}^{K_{\mathrm{TX}}} \sum_{l=1}^{K_{\mathrm{RX}}} \alpha_{k, \mathrm{TX}} \alpha_{l, \mathrm{RX}} \delta\left(\tau-\tau_{k, \mathrm{TX}}-\tau_{l, \mathrm{RX}}\right) \\
& +\sum_{k=1}^{K_{\mathrm{TX}}} \alpha_{k, \mathrm{TX}} \nu_{\mathrm{RX}}\left(\tau-\tau_{k, \mathrm{TX}}\right)+\sum_{l=1}^{K_{\mathrm{RX}}} \alpha_{l, \mathrm{RX}} \nu_{\mathrm{TX}}\left(\tau-\tau_{l, \mathrm{RX}}\right)
\end{aligned}
$$

$$
+\nu_{\mathrm{TXX}}(\tau) * \nu_{\mathrm{RX}}(\tau) .
$$

Here, the first term represents the deterministic part of the backscatter channel. The second and third terms are the convolution of the DM of the up-link channel with the deterministic components of the down-link channel, and vice versa. The last term constitutes the convolution of the DM of the upand down-link channels. In the following, we denote the sum of the last three terms of (3) that comprise the DM as

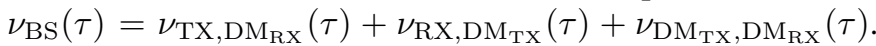
From (3) it is seen that the backscatter channel can be decomposed into a deterministic and a diffuse part, in the same way as the up- and down-links in (1).

With the quasi-stationarity, US assumption and that $\nu_{\mathrm{TX}}(\tau)$ and $\nu_{\mathrm{RX}}(\tau)$ are assumed to be independent, the PDP of the backscatter channel is the second central moment of the DM process

$$
\begin{aligned}
& S_{\nu, \mathrm{BS}}(\tau)=\mathbb{E}\left\{\nu_{\mathrm{BS}}(\tau) \nu_{\mathrm{BS}}^{*}(\tau)\right\} \\
& =\mathbb{E}\left\{\sum_{k=1}^{K_{\mathrm{TX}}} \sum_{k^{\prime}=1}^{K_{\mathrm{TX}}} \alpha_{k, \mathrm{TX}} \alpha_{k^{\prime}, \mathrm{TX}}^{*} \nu_{\mathrm{RX}}\left(\tau-\tau_{k, \mathrm{TX}}\right) \nu_{\mathrm{RX}}^{*}\left(\tau-\tau_{k^{\prime}, \mathrm{TX}}\right)\right\} \\
& +\mathbb{E}\left\{\sum_{l=1}^{K_{\mathrm{RX}}} \sum_{l^{\prime}=1}^{K_{\mathrm{RX}}} \alpha_{l, \mathrm{RX}} \alpha_{l^{\prime}, \mathrm{RX}}^{*} \nu_{\mathrm{TX}}\left(\tau-\tau_{l, \mathrm{RX}}\right) \nu_{\mathrm{TX}}^{*}\left(\tau-\tau_{l^{\prime}, \mathrm{RX}}\right)\right\} \\
& +\mathbb{E}\left\{\nu_{\mathrm{TX}}(\tau) * \nu_{\mathrm{RX}}(\tau)\left(\nu_{\mathrm{TX}}(\tau) * \nu_{\mathrm{RX}}(\tau)\right)^{*}\right\} .
\end{aligned}
$$

We assume a zero-mean Gaussian model for the DM, thus first and second moments give a complete description of the random process. The validity of the US assumption for a backscatter channel constituted by two US-channels has been proven in the appendix of [6], which leads to

$$
\begin{aligned}
& S_{\nu, \mathrm{BS}}(\tau)=\sum_{k=1}^{K_{\mathrm{TX}}}\left|\alpha_{k, \mathrm{TX}}\right|^{2} S_{\nu, \mathrm{RX}}\left(\tau-\tau_{k, \mathrm{TX}}\right) \\
& +\sum_{l=1}^{K_{\mathrm{RX}}}\left|\alpha_{l, \mathrm{RX}}\right|^{2} S_{\nu, \mathrm{TX}}\left(\tau-\tau_{l, \mathrm{RX}}\right)+S_{\nu, \mathrm{TX}}(\tau) * S_{\nu, \mathrm{RX}}(\tau) .
\end{aligned}
$$

In Fig. 2(a) the deterministic MPCs and the PDPs of the DM of some exemplary up- and down-link channels are shown. Fig. 2(b) shows the deterministic components $h_{\mathrm{BS}, \operatorname{det}}(\tau)$ and the individual terms of the PDP $S_{\nu, \mathrm{BS}}(\tau)$ of the backscatter channel.

\section{Transmitted Signal - Time-Reversal Processing}

We assume that the TX transmits a signal $s(t) \in \mathbb{C}$. On the one hand, this can be a single pulse $p(t)$ with pulse duration $T_{p}$, e.g. a commonly used root raised cosine pulse. Hence, the received signal represents the backscatter channel convolved with this pulse $p(t)$. On the other hand, the transmitted signal $s(t)$ can be a sum of complex weighted and time-shifted copies of this pulse $p(t)$ in order to obtain a TR signal.

TR processing is one promising candidate to overcome the degenerate pinhole nature of the backscatter channel, because it optimizes the link-budget between the TX and RX by focusing the energy onto the target. This is done by using the VAs, which are a geometric model for the deterministic 


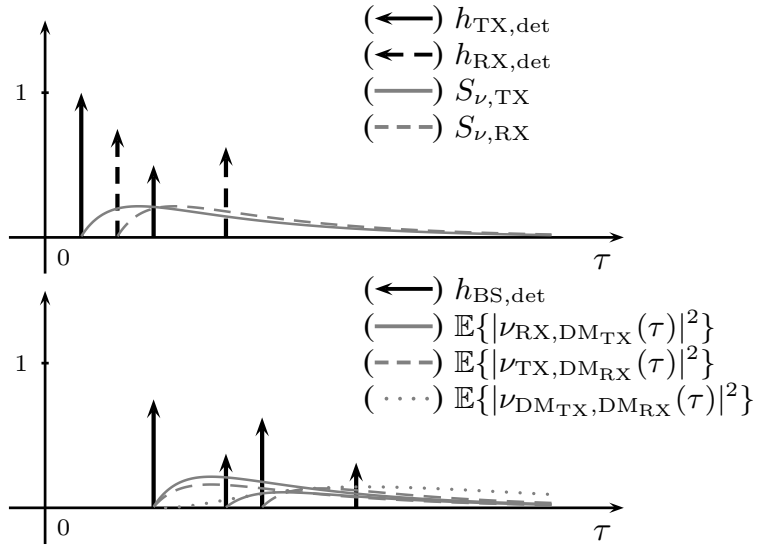

Fig. 2. (a) Up- and down-link channel. Solid lines denote the uplink channel, with deterministic components in black and the PDPs of the DM in gray. Dashed lines indicate the down-link channel. (b) Backscatter channel. Solid black lines denote the deterministic components. The gray lines indicate the different summands of the PDP.

MPCs of $h_{\mathrm{TX}}(\tau)$ in (1), as a virtual antenna array for spatial focusing. The TX signal becomes

$$
s(t)=\left[\sum_{k=1}^{\hat{K}_{\mathrm{TX}}} \hat{\alpha}_{k, \mathrm{TX}}^{*} \delta\left(t+\hat{\tau}_{k, \mathrm{TX}}\right)\right] * p(t)
$$

where $\left\{\hat{\alpha}_{k, \mathrm{TX}}, \hat{\tau}_{k, \mathrm{TX}}\right\}$ is the set of $\hat{K}_{\mathrm{TX}}(\tau)$ estimated MPC parameters of the up-link channel $h_{\mathrm{TX}}$ for a position $\hat{\mathbf{p}}$. The complex amplitudes are normalized s.t. $\int_{-\infty}^{\infty}|s(t)|^{2} \mathrm{~d} t=E_{\mathrm{TR}}$. The received signal at radar node RX can be obtained by the convolution of (3) with the transmit waveform (6) and AWGN $n(t)$ with a two-sided power spectral density of $N_{0} / 2$, given as

$$
\begin{aligned}
& r(t)=s(t) * h_{\mathrm{BS}}(t)+n(t) \\
& =\left[\sum_{k=1}^{K_{\mathrm{TX}}} \sum_{l=1}^{K_{\mathrm{RX}}} \sum_{k^{\prime}=1}^{\hat{K}_{\mathrm{TX}}} \alpha_{k, \mathrm{TX}} \alpha_{l, \mathrm{RX}} \hat{\alpha}_{k^{\prime}, \mathrm{TX}}^{*} \times \delta\left(t-\tau_{k, \mathrm{TX}}-\tau_{l, \mathrm{RX}}+\hat{\tau}_{k^{\prime}, \mathrm{TX}}\right)\right.
\end{aligned}
$$

The first term comprises deterministic components of the received signal $r(t)$, and the remaining terms constitute the DM arriving at the RX. Eq. (7) again shows that the channel described by the convolution of $h_{\mathrm{BS}}(\tau)$ and $h_{\mathrm{TR}}(\tau)$ can be decomposed into deterministic and diffuse parts. Assuming perfect TR parameters are available, (7) gives additional insights in the TR processing: First, the energy is concentrated on the deterministic MPCs of the down-link channel $h_{\ell, \operatorname{RX}}(\tau)$.
Second, again the structure of an equivalent deterministic channel and DM can be observed. The PDP of the received DM can be obtained as

$$
\begin{aligned}
& S_{\nu, \mathrm{TR}}(\tau)= \\
& =\sum_{k=1}^{K_{\mathrm{TX}}} \sum_{k^{\prime}=1}^{\hat{K}_{\mathrm{TX}}}\left|\alpha_{k, \mathrm{TX}}\right|^{2}\left|\hat{\alpha}_{k^{\prime}, \mathrm{TX}}\right|^{2} S_{\nu, \mathrm{RX}}\left(\tau-\tau_{k, \mathrm{TX}}+\hat{\tau}_{k^{\prime}, \mathrm{TX}}\right) \\
& +\sum_{l=1}^{K_{\mathrm{RX}}} \sum_{\hat{K}^{\prime}=1}^{\mathrm{TX}}\left|\alpha_{l, \mathrm{RX}}\right|^{2}\left|\hat{\alpha}_{k^{\prime}, \mathrm{TX}}\right|^{2} S_{\nu, \mathrm{TX}}\left(\tau-\tau_{l, \mathrm{RX}}+\hat{\tau}_{k^{\prime}, \mathrm{TX}}\right) \\
& +\sum_{k^{\prime}=1}^{\hat{K}_{\mathrm{TX}}}\left|\hat{\alpha}_{k^{\prime}, \mathrm{TX}}\right|^{2} \int_{-\infty}^{\infty} S_{\nu, \mathrm{TX}}(\lambda) S_{\nu, \mathrm{RX}}\left(\tau+\hat{\tau}_{k^{\prime}, \mathrm{TX}}-\lambda\right) \mathrm{d} \lambda .
\end{aligned}
$$

\section{ERRor Bound ON THE POSITION Estimation}

In this section, we derive the equivalent Fisher information matrix (EFIM) [1] for the target localization problem via the backscatter channel. The derivation and the notation follow closely [2] and [3]. Additionally, the influence of TR processing on the bound is analyzed.

\section{A. Problem Formulation}

Our goal is to estimate the position $\mathbf{p}$ of the target from the received signal $r(t)$ in the presence of DM and AWGN, using the TX and RX nodes at positions $\mathbf{p}_{1, \mathrm{TX}}$ and $\mathbf{p}_{1, \mathrm{RX}}$. With a-priori known floor-plan information, the TX and RX span two corresponding sets of VAs at positions $\left\{\mathbf{p}_{k, \mathrm{TX}}\right\}$ and $\left\{\mathbf{p}_{l, \mathrm{RX}}\right\}$. For the sake of simplicity, we introduce for the backscatter channel the equivalent propagation delays $\tau_{k, l}=\tau_{k, \mathrm{TX}}+\tau_{l, \mathrm{RX}}=\frac{1}{c}\left\|\mathbf{p}-\mathbf{p}_{k, \mathrm{TX}}\right\|+\frac{1}{c}\left\|\mathbf{p}-\mathbf{p}_{l, \mathrm{RX}}\right\|$, which are related to the geometry. Their corresponding complex amplitudes $\alpha_{k, l}=\alpha_{k, \mathrm{TX}} \alpha_{l, \mathrm{RX}}$ are nuisance parameters for the position estimation. We collect the delays $\tau_{k, l}$ and the real and imaginary parts of the amplitudes $\alpha_{k, l}$ in vectors $\tau, \boldsymbol{\alpha}^{\mathrm{R}}$, and $\boldsymbol{\alpha}^{\mathrm{I}}$ respectively.

The CRLB on the position error is a lower bound for the MSE of an unbiased estimator and is computed as the inverse of the Fisher information matrix (FIM) $\mathbf{J}(\boldsymbol{\theta})$ [11]. The vector of unknown parameters for position estimation is defined as $\boldsymbol{\theta}=\left[\mathbf{p}^{\mathrm{T}}\left(\boldsymbol{\alpha}^{\mathrm{R}}\right)^{\mathrm{T}}\left(\boldsymbol{\alpha}^{\mathrm{I}}\right)^{\mathrm{T}}\right]^{\mathrm{T}}$ (cf. [1]-[3]) and a transformed parameter vector related to the received signal $r(t)$ is $\boldsymbol{\psi}=$ $\left[\boldsymbol{\tau}^{\mathrm{T}}\left(\boldsymbol{\alpha}^{\mathrm{R}}\right)^{\mathrm{T}}\left(\boldsymbol{\alpha}^{\mathrm{I}}\right)^{\mathrm{T}}\right]^{\mathrm{T}}$. The FIM of the transformed parameter vector $\boldsymbol{\psi}$ is defined as

$$
\mathbf{J}(\boldsymbol{\psi})=\mathbb{E}_{\mathbf{r} \mid \boldsymbol{\psi}}\left\{-\frac{\partial^{2}}{\partial \boldsymbol{\psi} \partial \boldsymbol{\psi}} \ln \mathrm{p}(\mathbf{r} \mid \boldsymbol{\psi})\right\}
$$

where the observation vector $\mathbf{r}$ is obtained from the KarhunenLoéve expansion of the received signal $r(t)$ [11]. The FIM for position estimation is computed by applying the chain rule

$$
\mathbf{J}(\boldsymbol{\theta})=\mathbf{P} \mathbf{J}(\boldsymbol{\psi}) \mathbf{P}^{\mathrm{T}}
$$

where $\mathbf{P}=\partial \boldsymbol{\psi} / \partial \boldsymbol{\theta}$ is the the Jacobian of the transformation. 


\section{B. Likelihood Function of the Received Signal}

The likelihood function we use is adopted from [3]. Due to the fact that the $\mathrm{DM} \nu_{\mathrm{BS}}(\tau)$ is a colored non-stationary Gaussian noise process, a whitening operation has to be applied to the received signal $r(t)$ to obtain a tractable form. Given that the backscatter channel and the TR processed backscatter channel are both composed the same way as the channel used in [3], the framework to derive the likelihood function can be extended to backscatter channels. Using the Karhunen-Loéve expansion and integral equations [11], we can write

$$
\begin{aligned}
& \ln \mathrm{p}(\mathbf{r} \mid \boldsymbol{\psi}) \propto \\
& \frac{2}{N_{0}} \int_{0}^{T_{\mathrm{ob}}} \Re\left\{r(t) \sum_{k=1}^{K_{\mathrm{TX}}} \sum_{l=1}^{K_{\mathrm{RX}}} w_{k, l}^{2} \alpha_{k, l}^{*} s^{*}\left(t-\tau_{k, l}\right)\right\} \mathrm{d} t \\
& -\frac{1}{N_{0}} \int_{0}^{T_{\mathrm{ob}}}\left|\sum_{k=1}^{K_{\mathrm{TX}}} \sum_{l=1}^{K_{\mathrm{RX}}} w_{k, l} \alpha_{k, l} s\left(t-\tau_{k, l}\right)\right|^{2} \mathrm{~d} t
\end{aligned}
$$

where $T_{\mathrm{ob}}$ is the observation time and $w_{k, l}=$ $\sqrt{N_{0} /\left(N_{0}+T_{p} S_{\nu}\left(\tau_{k, l}\right)\right)}$ are the weighting factors accounting for DM. $S_{\nu}\left(\tau_{k, l}\right)$ denotes the PDP of the DM alone or with TR-processing. The term $T_{p} S_{\nu}\left(\tau_{k, l}\right)$ constitutes the inference power of the DM. The signal $s(t) \in \mathbb{C}$ either represents the TR waveform or the pulse waveform $p(t)$.

\section{EFIM and Position Error Bound (PEB)}

The PEB represents the CRLB on the position error at position $\mathbf{p}$ and is defined as

$$
\mathcal{P}(\mathbf{p}) \equiv \sqrt{\operatorname{tr}\left\{\left[\mathbf{J}(\boldsymbol{\theta})_{2 \times 2}\right]^{-1}\right\}}
$$

where $\operatorname{tr}\{\cdot\}$ is the trace of a square matrix. $\mathbf{J}(\boldsymbol{\theta})_{2 \times 2}$ is the upper left submatrix, which comprises the information on the position estimation and is called EFIM [1]. It leads to a reduction of the dimensionality of the FIM. The matrix $\mathbf{P}$ for the parameter transformation in (10) is

$$
\mathbf{P}=\left[\begin{array}{cc}
\mathbf{H}_{2 \times K_{\mathrm{TX}} K_{\mathrm{RX}}} & \mathbf{0}_{2 \times 2 K_{\mathrm{TX}} K_{\mathrm{RX}}} \\
\mathbf{0}_{2 K_{\mathrm{TX}} K_{\mathrm{RX}} \times 2} & \mathbf{I}_{2 K_{\mathrm{TX}} K_{\mathrm{RX}} \times 2 K_{\mathrm{TX}} K_{\mathrm{RX}}}
\end{array}\right]
$$

where $\mathbf{0}$ is the zero matrix, $\mathbf{I}$ denotes the identity matrix and $\mathbf{H}$ incorporates the geometry. The columns of $\mathbf{H}$ are of the form $-\frac{1}{c}\left[\cos \phi_{k}, \mathrm{TX}+\cos \phi_{l, \mathrm{RX}}, \sin \phi_{k, \mathrm{TX}}+\sin \phi_{l, \mathrm{RX}}\right]^{\mathrm{T}}$, where $\phi_{k, \mathrm{TX}}$ and $\phi_{l, \mathrm{RX}}$ are the angles between VAs of the TX- and RX-radar node and the target. For example on the TX side, this angle is defined as $\phi_{k, \mathrm{TX}}=\tan ^{-1}\left(\left(y-y_{k, \mathrm{TX}}\right) /\left(x-x_{k, \mathrm{TX}}\right)\right)$. The EFIM on the position error can be written as [3]

$$
\mathrm{EFIM} \equiv \mathbf{J}(\boldsymbol{\theta})_{2 \times 2}=\mathbf{H} \boldsymbol{\Lambda}_{\mathrm{A}} \mathbf{H}^{\mathrm{T}}-\mathbf{H} \boldsymbol{\Lambda}_{\mathrm{B}} \boldsymbol{\Lambda}_{\mathrm{C}}^{-1} \boldsymbol{\Lambda}_{\mathrm{B}}^{\mathrm{T}} \mathbf{H}^{\mathrm{T}}
$$

where the block matrices $\boldsymbol{\Lambda}_{\mathrm{A}}, \boldsymbol{\Lambda}_{\mathrm{B}}$ and $\boldsymbol{\Lambda}_{\mathrm{C}}$ are defined in the appendix. If there is no path overlap, which means that signals coming from different VAs do not intersect in the time-domain, $\boldsymbol{\Lambda}_{\mathrm{A}}$ is a diagonal matrix and $\boldsymbol{\Lambda}_{\mathrm{B}}$ is zero. According to [3], the EFIM can then be written in a canonical form a

$$
\mathbf{J}(\boldsymbol{\theta})_{2 \times 2}=\frac{8 \pi^{2} \beta^{2}}{c^{2}} \sum_{k=1}^{K_{\mathrm{TX}}} \sum_{l=1}^{K_{\mathrm{RX}}} \operatorname{SINR}_{k, l} \mathbf{J}_{\mathrm{r}}\left(\phi_{k}, \phi_{l}\right)
$$

where $\beta^{2}$ is the effective mean squared bandwidth of the pulse $p(t)$,

$$
\operatorname{SINR}_{k, l}=w_{k, l}^{2} \frac{\left|\alpha_{k, l}\right|^{2}}{N_{0}}=\frac{\left|\alpha_{k, l}\right|^{2}}{N_{0}+T_{p} S_{\nu}\left(\tau_{k, l}\right)}
$$

is the signal-to-interference-plus-noise ratio of the $k, l$-th backscatter MPC and

$$
\mathbf{J}_{\mathrm{r}}\left(\phi_{k}, \phi_{l}\right)=\left[\begin{array}{cc}
\mathrm{A}^{2} & \mathrm{AB} \\
\mathrm{AB} & \mathrm{B}^{2}
\end{array}\right]
$$

is the $2 \times 2$ ranging direction matrix accounting for the geometry, where $\mathrm{A}=\cos \phi_{k, \mathrm{TX}}+\cos \phi_{l, \mathrm{RX}}$ and $\mathrm{B}=$ $\sin \phi_{k, \mathrm{TX}}+\sin \phi_{l, \mathrm{RX}}$. Note that (15) in general does not hold for backscatter channels with TR processing, due to additional generated overlap of signal paths.

This analytical result was comprehensively analyzed for the single-channel MINT scenario in [3]. There, the main findings which also are evident in (15) are the following: First, any increase of the effective bandwidth decreases the PEB. Second, each additional VA increases the EFIM and consequently decreases the PEB, and third, the gain of each $\mathrm{VA}$ is determined by the corresponding SINR.

\section{Influence of TR Processing on the Position Error Bound}

One impact of TR processing on the CRLB is that the weights $w_{k, l}$ accounting for the DM change according to the PDP $S_{\nu, \mathrm{TR}}$. Furthermore, TR processing influences the signal correlation function, which appears in the block matrices of the EFIM (c.f. appendix), the following way

$$
\begin{aligned}
& \mathrm{R}_{s}\left(\tau_{k, l}-\tau_{k^{\prime}, l^{\prime}}\right)=\int_{-\infty}^{\infty} s\left(t-\tau_{k, l}\right) s\left(t-\tau_{k^{\prime}, l^{\prime}}\right) \mathrm{d} t \\
&= \int_{-\infty}^{\infty} \sum_{m=1}^{\hat{K}_{\mathrm{TX}}} \sum_{m^{\prime}=1}^{\hat{K}_{\mathrm{TX}}}\left|\hat{\alpha}_{m, \mathrm{TX}}\right|^{2}\left|\hat{\alpha}_{m^{\prime}, \mathrm{TX}}\right|^{2} \\
&= \sum_{m=1} \sum_{m^{\prime}=1}^{\hat{K}_{\mathrm{TX}}}\left|\hat{\alpha}_{m, \mathrm{TX}}\right|^{2}\left|\hat{\alpha}_{m^{\prime}, \mathrm{TX}}\right|^{2} \\
& \mathrm{R}_{p}\left(\left(\tau_{k, l}-\hat{\tau}_{m, \mathrm{TX}}\right)-\left(\tau_{k^{\prime}, l^{\prime}}-\hat{\tau}_{m^{\prime}, \mathrm{TX}}\right)\right) .
\end{aligned}
$$

where $\mathrm{R}_{p}\left(\tau_{k, l}-\tau_{k^{\prime}, l^{\prime}}\right)=\int_{-\infty}^{\infty} p\left(t-\tau_{k, l}\right) p\left(t-\tau_{k^{\prime}, l^{\prime}}\right) \mathrm{d} t$ is the ACF of the transmitted pulse $p(t)$. Eq. (18) illustrates the additional generated path overlap by TR processing.

\section{RESUlts}

1) Simulation Setup: A computational analysis has been performed for the scenario illustrated in Fig. 1 for a target that moves along a trajectory consisting of 24 target positions. The TX radar node is located at position $\mathbf{p}_{1, \mathrm{TX}}=[8,7.5]^{\mathrm{T}}$ and the RX radar node at position $\mathbf{p}_{1, \mathrm{RX}}=[2,6]^{\mathrm{T}}$. Deterministic MPCs of the up- and down-link channels $h_{\mathrm{TX}}(\tau)$ and $h_{\mathrm{RX}}(\tau)$ have been generated using VAs for first- and second-order reflections and the LOS components. A similar path-loss model has been used for the MPC gain as in [3], assuming a carrier frequency of $7 \mathrm{GHz}$ and adding $3 \mathrm{~dB}$ of attenuation for each reflection order. The transmitted pulse $p(t)$ is modeled 


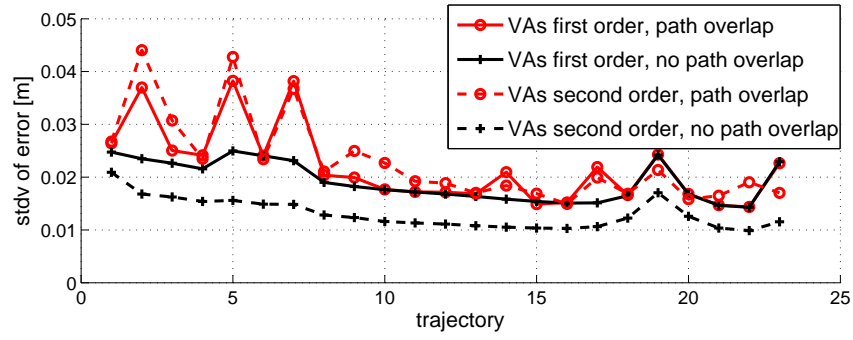

Fig. 3. PEB along a trajectory for backscatter channel for VA order 1 and 2 , with and without path overlap, $T_{p}=0.5 \mathrm{~ns}$.

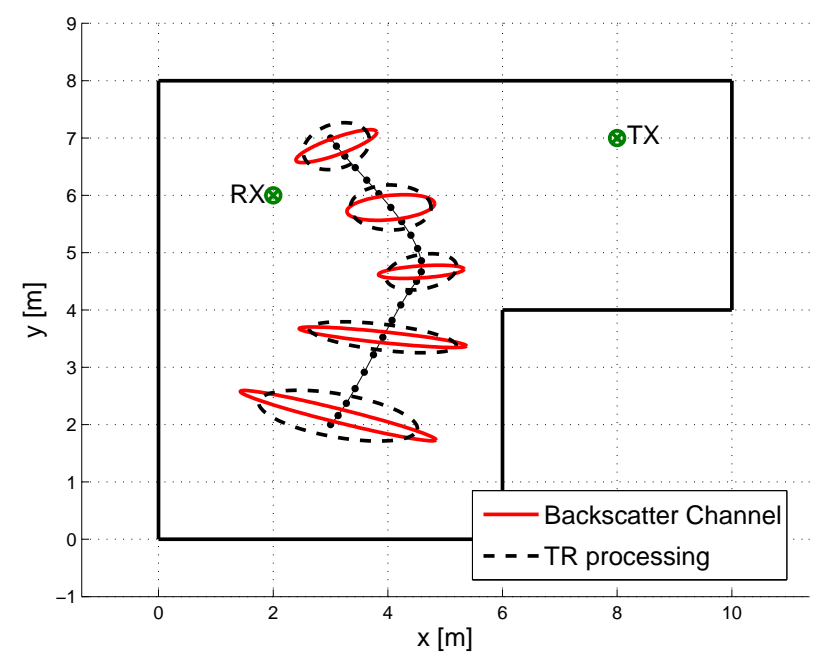

Fig. 4. Simulation scenario. Position error ellipses of the forty-fold standard deviation, $T_{p}=0.5 \mathrm{~ns}$.

as root raised cosine pulse with roll-off factor $\beta_{\text {roll }}=0.6$ and pulse duration of $T_{p}=0.5 \mathrm{~ns}$ corresponding to a bandwidth of $2 \mathrm{GHz}$. The PDPs of the diffuse part of both channels are modeled as double-exponential functions cf. [12, (9)]. Their parameters are the total power of the diffuse multipath $\Omega_{1}=1.16 \times 10^{-6}$, the decay time $\gamma_{1}=20 \mathrm{~ns}$, the rise time $\gamma_{\text {rise }}=5 \mathrm{~ns}$ and the shape parameter $\chi=0.98$, which were kept fixed over the entire trajectory. However, due to the concatenation of the up- and down-link channels, the resulting PDP of the DM for the backscatter channel depends on the deterministic channel parts, and thus on the target position. The SNR of DM varies along the trajectory between $25 \mathrm{~dB}$ and $31 \mathrm{~dB}$. The SINR of the LOS component at the first trajectory position $\mathbf{p}=[3,2]$ is $21 \mathrm{~dB}$ for the backscatter channel and $15 \mathrm{~dB}$ for the backscatter channel with TR processing.

2) CRLB and Influence of TR Processing: We first analyze the CRLB for backscatter channels without TR processing. Due to the fact that in a backscatter channel more MPCs appear than in a conventional channel, path-overlap is more probable, which causes stronger degradation of the PEB. This is illustrated in Fig. 3, where the PEB is computed from the complete EFIM (14) and from the canonical form of the EFIM that neglects path-overlap. First-order reflections (solid lines) and second-order reflections (dashed lines) are analyzed. We

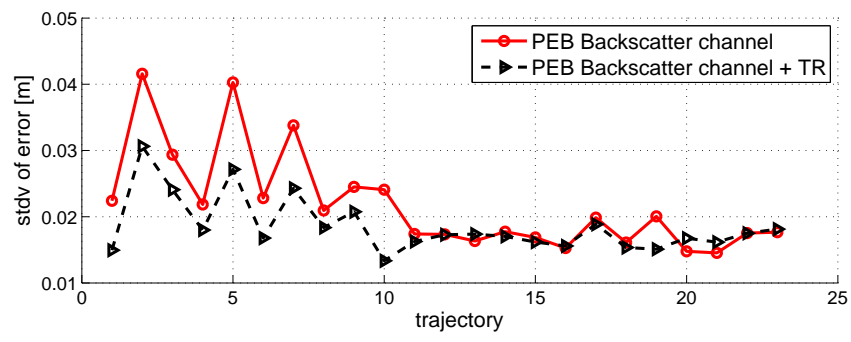

(a) LOS, VA order 1 for TR processing

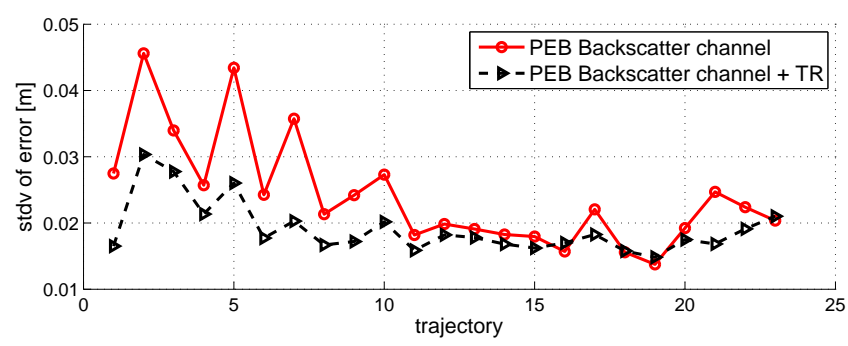

(b) NLOS, VA order 1 for TR processing

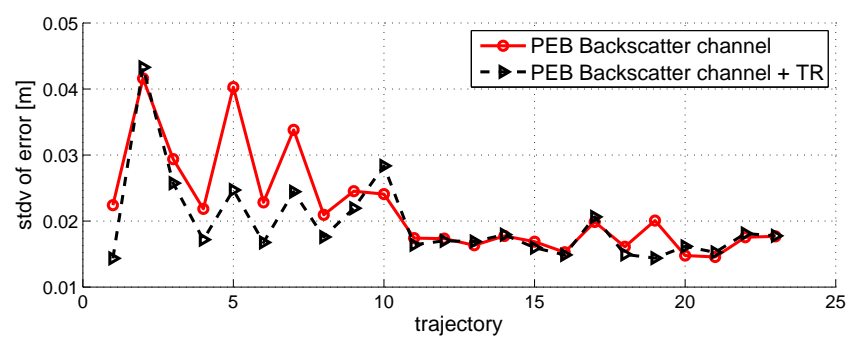

(c) LOS, VA order 2 for TR processing

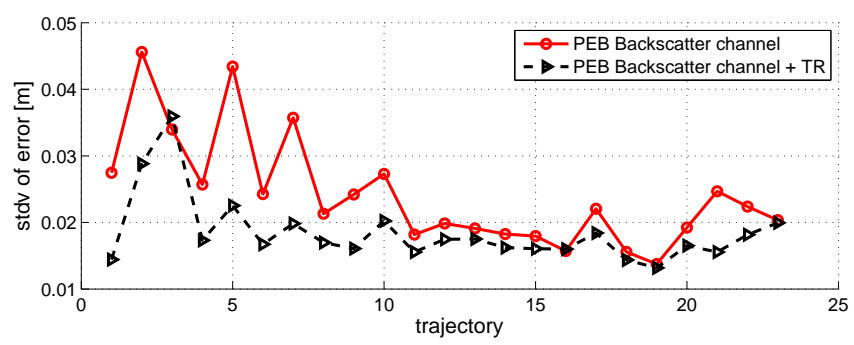

(d) NLOS, VA order 2 for TR processing

Fig. 5. PEB along a trajectory for LOS and NLOS scenarios with VA order of 1 for TR processing (path overlap considered), $T_{p}=0.5 \mathrm{~ns}$.

can see that the PEB neglecting path overlap is decreased with increasing order of MPCs, since more information can be exploited. On the other hand, the PEB considering pathoverlap is partly increased since path-overlap makes this information unusable.

Fig. 4 shows the impact of TR processing using VA order of 2. Position error ellipses are given in terms of the fortyfold standard deviation for several trajectory positions. Red (solid) stands for the backscatter channel alone and black (dashed) includes TR processing. One can first see that the error depends on the geometry. It is lower in the direction of the radar nodes, because MPCs from other directions are more 
impaired by DM. An interesting fact is that TR processing partly levels this imbalance. It improves the information from reflected MPCs, while reducing information from the LOS component that gets affected by DM more significantly, due which is a fundamental requirement for a cognitive radar to gain control over the environment sensing in its perceptionaction cycle [13]. The overall PEB is decreased by TR processing for most positions, but the gain depends strongly on the geometry.

Figs. 5(a)-(d) show the PEB of the backscatter channel with TR processing along the trajectory for the LOS and NLOS cases (path overlap considered), where the latter means that the first component of the TX channel has been set to zero. Figs. 5(a) and (b) illustrate TR processing with MPCs coming from VAs of first-order. One can see that TR processing results in a performance gain, especially for NLOS scenarios. As Figs. 5(c) and (d) show, the inclusion of second-order reflections in TR parameter set does not automatically yields in a performance gain. This can be explained by the fact that the impairment of additional DM is higher than the gain caused by energy focusing. The results illustrate again that the performance gain through TR processing is strongly dependent on the geometry of the room.

\section{CONCLUSIONS}

Using a channel model that explicitly models the diffuse multipath, a unified likelihood model for the localization on backscatter channels has been introduced. The impact of TR processing can be evaluated as well. Results show the detrimental effect of path overlap and DM in backscatter channels. Using geometrically modeled deterministic MPCs for TR processing does not automatically imply large performance gains. The special structure of DM in the backscatter channel suggest a careful usage of a subset of the uplink paths for TR, which is supported by our derivations and results. TR processing yields a closed control loop for optimal sensing of the environment as it is required in a cognitive radar system. The CRLB can be used as feedback information as it quantifies the performance gain.

\section{APPENDIX}

\section{A. Derivation of the Subblocks of the FIM}

The FIM $\mathbf{J}(\boldsymbol{\psi})$ of the transformed parameter vector $\boldsymbol{\psi}=$ $\left[\tau^{\mathrm{T}}\left(\boldsymbol{\alpha}^{\mathrm{R}}\right)^{\mathrm{T}}\left(\boldsymbol{\alpha}^{\mathrm{I}}\right)^{\mathrm{T}}\right]^{\mathrm{T}}$ can be calculated from (9) the following way

$$
\mathbf{J}(\boldsymbol{\psi})=\left[\begin{array}{ccc}
\boldsymbol{\Lambda}_{\mathrm{A}} & \boldsymbol{\Lambda}_{\mathrm{B}}^{\mathrm{R}} & \boldsymbol{\Lambda}_{\mathrm{B}}^{\mathrm{I}} \\
\left(\boldsymbol{\Lambda}_{\mathrm{B}}^{\mathrm{R}}\right)^{\mathrm{T}} & \boldsymbol{\Lambda}_{\mathrm{C}}^{\prime} & \boldsymbol{\Lambda}_{\mathrm{C}}^{\prime \prime} \\
\left(\boldsymbol{\Lambda}_{\mathrm{B}}^{\mathrm{I}}\right)^{\mathrm{T}} & \boldsymbol{\Lambda}_{\mathrm{C}}^{\prime \prime} & \boldsymbol{\Lambda}_{\mathrm{C}}^{\prime}
\end{array}\right]_{3 K_{\mathrm{TX}} K_{\mathrm{RX}} \times 3 K_{\mathrm{TX}} K_{\mathrm{RX}}}
$$

where $\boldsymbol{\Lambda}_{\mathrm{B}}=\left[\begin{array}{ll}\boldsymbol{\Lambda}_{\mathrm{B}}^{\mathrm{R}} & \boldsymbol{\Lambda}_{\mathrm{B}}^{\mathrm{I}}\end{array}\right]$ and $\boldsymbol{\Lambda}_{\mathrm{C}}=\left[\begin{array}{lll}\boldsymbol{\Lambda}_{\mathrm{C}}^{\prime} & \boldsymbol{\Lambda}_{\mathrm{C}}^{\prime \prime} ; \boldsymbol{\Lambda}_{\mathrm{C}}^{\prime \prime} & \boldsymbol{\Lambda}_{\mathrm{C}}^{\prime}\end{array}\right]$. The sub-blocks are derived as

$$
\begin{aligned}
{\left[\boldsymbol{\Lambda}_{\mathrm{A}}\right]_{k l, k^{\prime} l^{\prime}} } & =\mathbb{E}_{\mathbf{r} \mid \boldsymbol{\psi}}\left\{\frac{\partial^{2} \ln \mathrm{p}(\mathbf{r} \mid \boldsymbol{\psi})}{\partial \tau_{k, l} \partial \tau_{k^{\prime}, l^{\prime}}}\right\} \\
& =\frac{2}{N_{0}} w_{k, l} w_{k^{\prime}, l^{\prime}} \Re\left\{\alpha_{k, l} \alpha_{k^{\prime}, l^{\prime}}^{*} \frac{\partial^{2} \mathrm{R}_{s}\left(\tau_{k, l}-\tau_{k^{\prime}, l^{\prime}}\right)}{\partial \tau_{k, l} \partial \tau_{k^{\prime}, l^{\prime}}}\right\}
\end{aligned}
$$

$$
\begin{aligned}
{\left[\boldsymbol{\Lambda}_{\mathrm{B}}^{\mathrm{R}}\right]_{k l, k^{\prime} l^{\prime}} } & =\mathbb{E}_{\mathbf{r} \mid \boldsymbol{\psi}}\left\{\frac{\partial^{2} \ln \mathrm{p}(\mathbf{r} \mid \boldsymbol{\psi})}{\partial \tau_{k, l} \partial \alpha_{k^{\prime}, l^{\prime}}^{\mathrm{R}}}\right\} \\
& =\frac{2}{N_{0}} w_{k, l} w_{k^{\prime}, l^{\prime}} \Re\left\{\alpha_{k, l} \frac{\partial \mathrm{R}_{s}\left(\tau_{k, l}-\tau_{k^{\prime}, l^{\prime}}\right)}{\partial \tau_{k, l}}\right\} \\
{\left[\boldsymbol{\Lambda}_{\mathrm{B}}^{\mathrm{I}}\right]_{k l, k^{\prime} l^{\prime}} } & =\mathbb{E}_{\mathbf{r} \mid \boldsymbol{\psi}}\left\{\frac{\partial^{2} \ln \mathrm{p}(\mathbf{r} \mid \boldsymbol{\psi})}{\partial \tau_{k, l} \partial \alpha_{k^{\prime}, l^{\prime}}^{\mathrm{I}}}\right\} \\
& =\frac{2}{N_{0}} w_{k, l} w_{k^{\prime}, l^{\prime}} \Im\left\{\alpha_{k, l} \frac{\partial \mathrm{R}_{s}\left(\tau_{k, l}-\tau_{k^{\prime}, l^{\prime}}\right)}{\partial \tau_{k, l}}\right\} \\
{\left[\boldsymbol{\Lambda}_{\mathrm{C}}^{\prime}\right]_{k l, k^{\prime} l^{\prime}} } & =\mathbb{E}_{\mathbf{r} \mid \boldsymbol{\psi}}\left\{\frac{\partial^{2} \ln \mathrm{p}(\mathbf{r} \mid \boldsymbol{\psi})}{\partial \alpha_{k, l}^{\mathrm{R}} \partial \alpha_{k^{\prime}, l^{\prime}}^{\mathrm{R}}}\right\}=\mathbb{E}_{\mathbf{r} \mid \boldsymbol{\psi}}\left\{\frac{\partial^{2} \ln \mathrm{p}(\mathbf{r} \mid \boldsymbol{\psi})}{\partial \alpha_{k, l}^{\mathrm{I}} \partial \alpha_{k^{\prime}, l^{\prime}}^{\mathrm{I}}}\right\} \\
& =\frac{2}{N_{0}} w_{k, l} w_{k^{\prime}, l^{\prime}} \Re\left\{\mathrm{R}_{s}\left(\tau_{k, l}-\tau_{k^{\prime}, l^{\prime}}\right)\right\} \\
{\left[\boldsymbol{\Lambda}_{\mathrm{C}}^{\prime \prime}\right]_{k l, k^{\prime} l^{\prime}} } & =\mathbb{E}_{\mathbf{r} \mid \boldsymbol{\psi}}\left\{\frac{\partial^{2} \ln \mathrm{p}(\mathbf{r} \mid \boldsymbol{\psi})}{\partial \alpha_{k, l}^{\mathrm{R}} \partial \alpha_{k^{\prime}, l^{\prime}}^{\mathrm{I}}}\right\} \\
& =\frac{2}{N_{0}} w_{k, l} w_{k^{\prime}, l^{\prime}} \Im\left\{\mathrm{R}_{s}\left(\tau_{k, l}-\tau_{k^{\prime}, l^{\prime}}\right)\right\}
\end{aligned}
$$

where $\mathrm{R}_{s}\left(\tau_{k, l}-\tau_{k^{\prime}, l^{\prime}}\right)=\int_{-\infty}^{\infty} s\left(t-\tau_{k, l}\right) s\left(t-\tau_{k^{\prime}, l^{\prime}}\right) \mathrm{d} t$ is the signal correlation function.

\section{REFERENCES}

[1] Y. Shen and M. Win, "Fundamental limits of wideband localization; part I: A general framework," Information Theory, IEEE Transactions on, vol. 56, no. 10, pp. $4956-4980$, Oct. 2010.

[2] H. Godrich, V. Chiriac, A. Haimovich, and R. Blum, "Target tracking in mimo radar systems: Techniques and performance analysis," in Radar Conference, 2010 IEEE, 2010, pp. 1111-1116.

[3] K. Witrisal and P. Meissner, "Performance bounds for multipath-assisted indoor navigation and tracking (MINT)," in Communications (ICC), 2012 IEEE International Conference on, June 2012, pp. $4321-4325$.

[4] P. Meissner and K. Witrisal, "Analysis of position-related information in measured UWB indoor channels," in Antennas and Propagation (EUCAP), 2012 6th European Conference on, March 2012, pp. 6 -10.

[5] P. Meissner, E. Leitinger, M. Froehle, and K. Witrisal, "Accurate and robust indoor localization systems using ultra-wideband signals," in European Conference on Navigation (ENC), Vienna, 2013.

[6] D. Arnitz, U. Muehlmann, and K. Witrisal, "Wideband characterization of backscatter channels: Derivations and theoretical background," $A n$ tennas and Propagation, IEEE Transactions on, vol. 60, no. 1, pp. 257 -266 , Jan. 2012.

[7] T. Strohmer, M. Emami, J. Hansen, G. Papanicolaou, and A. Paulraj, "Application of time-reversal with mmse equalizer to UWB communications," in Global Telecommunications Conference, 2004. GLOBECOM '04. IEEE, vol. 5, Nov. 2004, pp. 3123 - 3127 Vol.5.

[8] N. Guo, B. Sadler, and R. Qiu, "Reduced-complexity uwb time-reversal techniques and experimental results," Wireless Communications, IEEE Transactions on, vol. 6, no. 12, pp. 4221 -4226, Dec. 2007.

[9] K. Witrisal, E. Leitinger, P. Meissner, and D. Arnitz, "Cognitive radar for the localization of RFID transponders in dense multipath environments," in Radar (RadarCon), 2013 IEEE International Conference on, April 2013.

[10] A. Molisch, "Ultra-wide-band propagation channels," Proceedings of the IEEE, vol. 97, no. 2, pp. 353 -371, Feb. 2009.

[11] H. L. Van Trees, Detection, Estimation and Modulation, Part I. Wiley Press, 1968.

[12] J. Karedal, S. Wyne, P. Almers, F. Tufvesson, and A. Molisch, "A measurement-based statistical model for industrial ultra-wideband channels," Wireless Communications, IEEE Transactions on, vol. 6, no. 8, pp. 3028-3037, Aug.

[13] S. Haykin, Y. Xue, and P. Setoodeh, "Cognitive radar: Step toward bridging the gap between neuroscience and engineering," Proceedings of the IEEE, vol. 100, no. 11, pp. 3102 -3130, nov. 2012. 